\title{
Plant Immune System: Crosstalk Between Responses to Biotic and Abiotic Stresses the Missing Link in Understanding Plant Defence
}

\author{
Naghmeh Nejat* and Nitin Mantri* \\ School of Science, Health Innovations Research \\ Institute, RMIT University, Melbourne, Victoria, \\ Australia
}

*Corresponding authors: nitin.mantri@rmit.edu.au; nanejat@gmail.com;

DOI: http://dx.doi.org/10.21775/cimb.023.001

\begin{abstract}
Environmental pollution, global warming and climate change exacerbate the impact of biotic and abiotic stresses on plant growth and yield. Plants have evolved sophisticated defence network, also called innate immune system, in response to everchanging environmental conditions. Significant progress has been made in identifying the key stress-inducible genes associated with defence response to single stressors. However, relatively little information is available on the signaling crosstalk in response to combined biotic/abiotic stresses. Recent evidence highlights the complex nature of interactions between biotic and abiotic stress responses, significant aberrant signaling crosstalk in response to combined stresses and a degree of overlap, but unique response to each environmental stimulus. Further, the results of simultaneous combined biotic and abiotic stress studies indicate that abiotic stresses particularly heat and drought enhance plant susceptibility to plant pathogens. It is noteworthy that global climate change is predicted to have a negative impact on biotic stress resistance in plants. Therefore, it is vital to conduct plant transcriptome analysis in response to combined stresses to identify general or multiple stress- and pathogen-specific genes that confer multiple stress tolerance in plants under climate change. Here, we discuss the recent advances in our understanding of the molecular mechanisms of crosstalk in response to biotic and abiotic stresses. Pinpointing both, common and specific components of the signaling crosstalk in plants, allows
\end{abstract}

identification of new targets and development of novel methods to combat biotic and abiotic stresses under global climate change.

\section{Introduction}

Biotic and abiotic stresses, as a part of the natural ecosystem, seriously impact crop productivity and threaten global food security. Plants in their natural habitats are persistently and simultaneously confronted with a range of biotic (e.g. biotrophic and necrotrophic fungi, bacteria, phytoplasmas, oomycetes and nematodes, and non-cellular pathogens i.e. viruses and viroids) and abiotic stress factors (such as heat, cold, drought, salinity, mechanical wounds, high light intensity, freezing, heavy metals and metalloids; Mantri et al. 2014).

Plants have evolved a plethora of complex immune response pathways which enable them as sessile organisms to survive not only specific stresses but also a combination of stresses (Nejat et al. 2015). Plant-biotic and/or abiotic stress interactions trigger a wide range of defence reactions at molecular and cellular levels to ensure that plant cells are insulated from the challenges they encounter (Kim et al. 2014) (Figure 1).

A sophisticated defence network is activated to orchestrate transcriptional reprogramming using a two-tiered defence approach (Dodds and Rathjen 2010; Tsuda and Katagiri 2010). Pathogen/microbe associated molecular patterns (PAMP/MAPM)triggered immunity $(\mathrm{PTI})$ is the first line of defence. It is a general and non-specific defence response and provides basal resistance not only against entire classes of microbial pathogens but also abiotic stresses. PTI constitutes the inducible defence through an array of early cellular responses comprising of ion flux across the membrane, production of reactive oxygen species (ROS) and mitogen-activated protein kinase (MAPK) cascades phosphorylation and long-term later responses that induce callose deposition (Nakagami et al. 2005; Dodds and Rathjen 2010; Tsuda and Katagiri 2010; 


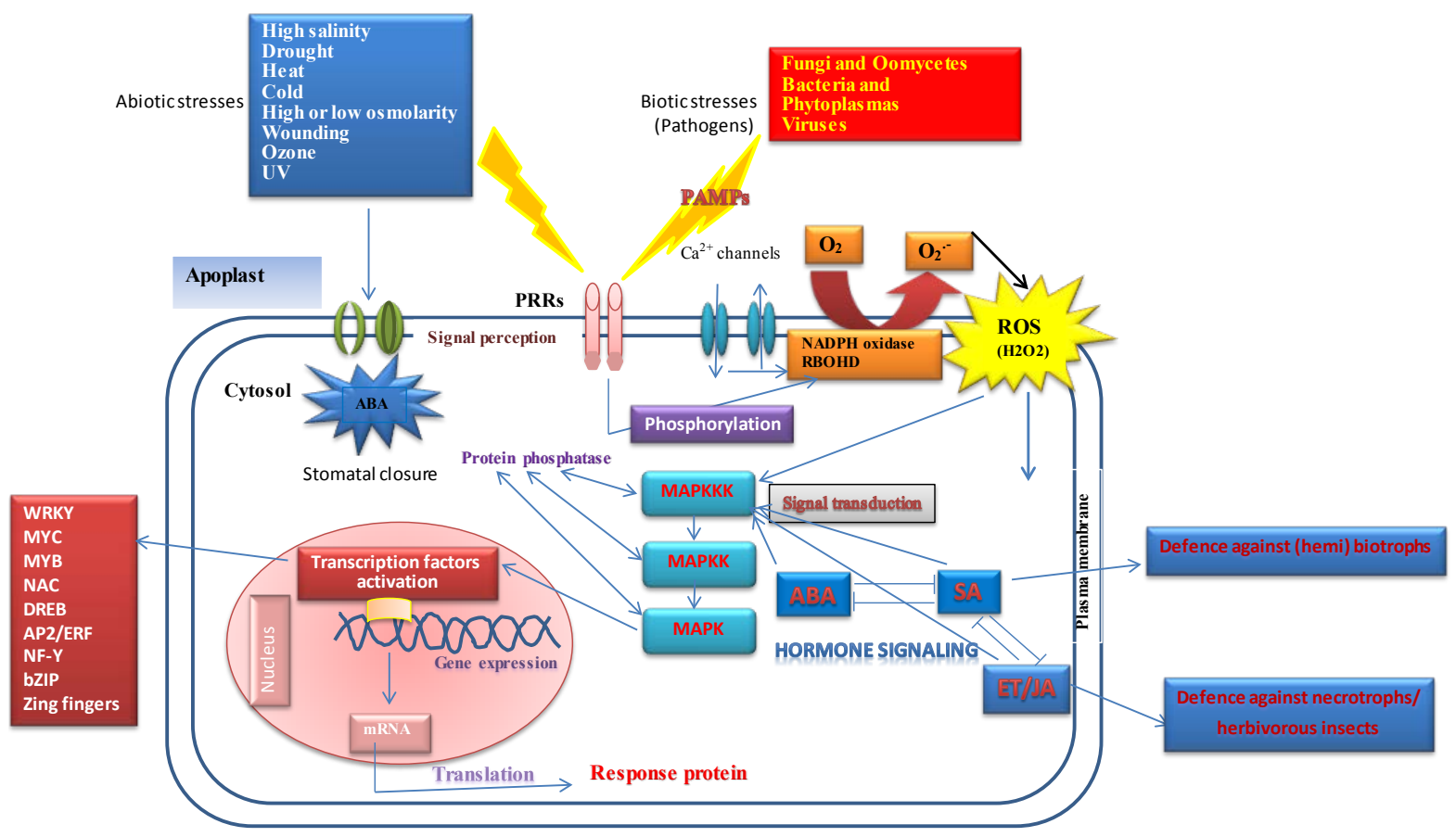

Figure 1. The overall model of plant immunity in response to biotic and abiotic stresses. Perception of pathogen-associated molecular patterns (PAMPs), sensing abiotic stress and/or any extracellular signals by surface-localised pattern recognition receptors (PRRs) trigger phosphorylation of the RBOHD (respiratory burst oxidase-D) and activate the NADPH oxidase RBOHD for rapid production of reactive oxygen species (ROS) in calcium-independent or dependent manner, which subsequently trigger mitogen-activated protein kinase (MAPK) phosphorylation as general defence response. MAPK transduce extracellular signals to nucleus leading to activation of transcription factors that regulate immunity gene expression. There is antagonistic and synergistic crosstalk between hormone signal transduction pathways in response to various attackers. ABA: abscisic acid; SA: salicylic acid; JA: jasmonic acid; ET: ethylene.

Schwessinger et al. 2015). Defence-related phytohormones comprise salicylic acid (SA), jasmonic acid (JA), ethylene (ET) and abscisic acid (ABA) as the core immune signaling components. They are produced in response to both, biotic and abiotic stresses (Dodds and Rathjen 2010; Tsuda and Katagiri 2010). Among those, ROS, MAPkinase cascades and hormone signalling pathways are of paramount importance as they lead the defence responses to biotic and abiotic stresses (Nakagami et al. 2005; Fujita et al. 2006; Atkinson and Urwin 2012; Kissoudis et al. 2014; Perez and Brown 2014; Schwessinger et al. 2015) (Figure 2). Therefore, the authors propose that PTI which is activated either by PAMP or MAPM is the "stresstriggered immunity" as it has evolutionary conserved molecular signatures across similar types of microbes or abiotic stresses.

The effector-triggered immunity $(\mathrm{ETI})$ is the second, prolonged and robust layer of plant immune response that is specific to the pathogen infection. It is activated by resistance $(R)$ genes when pathogen virulence factors, called effectors, are released into plant cells (Cui et al 2015). If the first line of inducible non-specific defence, so-called PTI, is successfully overcome by pathogens through suppression of PTI response, then the second line of plant immunity is activated to produce defence proteins that cause programmed cell death. This is also referred to as the hypersensitive response (HR) (Thomma et al. 2011).

Hence, after stress recognition, the immune system triggers a wide range of defence mechanisms to orchestrate transcriptional reprogramming through receptor proteins, signal transduction cascades, kinase cascades, reactive oxygen species, hormone signaling pathways, heat shock proteins, and transcription factors to protect plants against different attackers (Figure 1). However, recent advances suggest significant aberrant signaling 


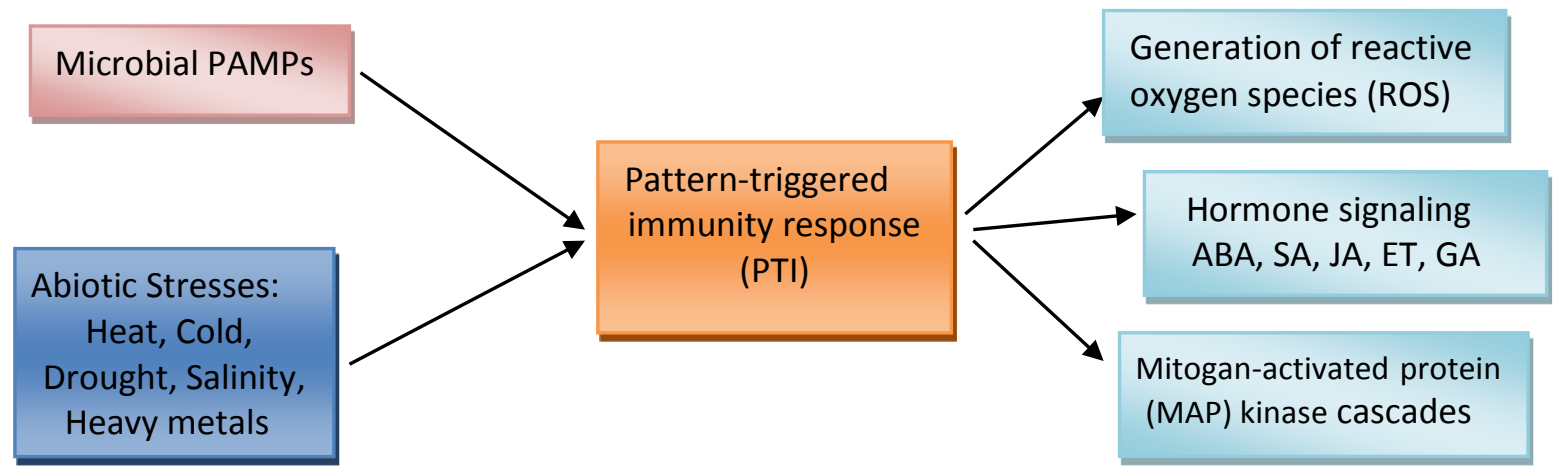

Figure 2. Activation of the first line of defence in a non-specific approach. ABA: Abscisic acid; SA: Salicylic acid; JA: Jasmonic acid; ET: Ethylene; GA: Gibberellins.

crosstalk in plant immune response to a combination of biotic and abiotic stresses compared with an individual stress (Atkinson et al. 2013; Prasch et al. 2013; Suzuki et al. 2014). In this review, we discuss the major factors affecting signaling pathways in response to single and combined stresses, their common and overlapping responses, and the crosstalk between signaling pathways in response to biotic and abiotic stresses.

\section{Crosstalk between responses to environmental stimuli in the post-genome era}

Plant responses to biotic and abiotic stresses involve complex signaling pathways. Increasing evidence suggest that there is significant overlap and several defence genes are commonly involved in response to multiple biotic and abiotic stresses as shared or general stress-responsive genes (Table 1) (Massa et al. 2013; Mantri et al., 2010; Narsai et al. 2013; Shaik and Ramakrishna 2013; Sham et al. 2014,2015; Zhang et al. 2016). Nevertheless, in response to combination of stresses, plants orchestrate extensive transcriptional reprogramming and exhibit a unique program of transcript response that is not similar to that of either stresses individually, although there is significant overlap in response pathways to combined environmental stresses (Mantri et al. 2010; Atkinson 2011; Prasch and Sonnewald 2013; Rasmussen et al. 2013; Sham et al. 2015). Several studies revealed crosstalk between responses to combined stress treatment synergistically or antagonistically. Of note, abiotic stresses have a negative impact on biotic stress resistance and can lead to enhanced plant susceptibility to biotic stresses (Wang et al. 2009;
Atkinson 2011; Prasch and Sonnewald 2013; Sewelam et al. 2014; Kissoudis et al. 2015).

Study of the molecular response of $A$. thaliana to the concurrent drought stress and plant-parasitic nematode, Heterodera schachtii, infection using microarrays revealed that drought stress increased the susceptibility of $A$. thaliana to nematode infection. Moreover, induction patterns of differentially expressed genes following these stress treatments displayed not only a specific response to each stress but also a particular response that was uniquely activated by the combination of biotic/ abiotic stress (Atkinson 2011).

Similarly, combined heat and drought stress increased the susceptibility of Arabidopsis plants to Turnip mosaic virus infections through suppression of defence responses to the biotic stress (Prasch and Sonnewald, 2013). Further, salt stress significantly increased the susceptibility of tomato plants to powdery mildew (Kissoudis et al. 2015).

In 2010, Mantri et al. analysed the chickpea transcriptome in response to drought, cold, highsalinity and the necrotrophic fungal pathogen Ascochyta rabiei using 768-feature boutique microarray. Their results showed that $A$. rabiei and high salinity which were both studied at the seedling stage, shared the highest number of deferentially expressed transcripts and to a lesser extent between $A$. rabiei and cold, and $A$. rabiei and drought which were studied at flowering stage. Further, 51 transcripts were differentially expressed in the shoot tissues in response to $A$. rabiei, of which 21 transcripts were commonly differentially 
Table 1. Key genes responsive to biotic and abiotic stresses in various plant species.

\begin{tabular}{|c|c|c|c|c|}
\hline Gene & Plant species & Stressors & Function & References \\
\hline HSPs & A. Thaliana & 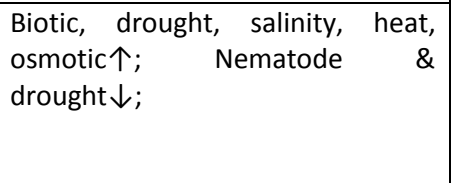 & $\begin{array}{l}\text { stabilize } \\
\text { proteins }\end{array}$ & $\begin{array}{l}\text { Sham et al. 2014; 2015; } \\
\text { Ma et al. 2007; } \\
\text { Rasmussen et al. 2013; } \\
\text { Sewelam et al. 2014; } \\
\text { Atkinson 2011; }\end{array}$ \\
\hline ABA & A. Thaliana & $\begin{array}{l}\text { Drought, cold, salinity, cold, } \\
\text { osmotic } \uparrow\end{array}$ & $\begin{array}{l}\text { Defence against } \\
\text { abiotic stresses }\end{array}$ & $\begin{array}{l}\text { Seki et al. 2002; Kreps et } \\
\text { al. } 2002\end{array}$ \\
\hline $\begin{array}{l}\text { Ethylene } \\
\text { biosynthesis genes }\end{array}$ & A. Thaliana & Drought, cold, salinity $\uparrow$ & Defence signals & Seki et al. 2002 \\
\hline $\begin{array}{l}\text { Senescence-related } \\
\text { genes }\end{array}$ & A. Thaliana & $\begin{array}{l}\text { Nematode, virus, heat \& } \\
\text { drought } \uparrow ;\end{array}$ & Defence signals & $\begin{array}{l}\text { Atkinson 2011; Prasch \& } \\
\text { Sonnewald 2013; Kreps } \\
\text { et al. } 2002\end{array}$ \\
\hline PR proteins & A. Thaliana & Virus \& heat $\downarrow$ & Resistance & $\begin{array}{l}\text { Prasch \& Sonnewald } \\
\text { 2013; }\end{array}$ \\
\hline $\begin{array}{l}\text { Photosynthesis- } \\
\text { related genes }\end{array}$ & $\begin{array}{l}\text { C. arietinum, } \\
\text { A. Thaliana, } O . \\
\text { sativa }\end{array}$ & $\begin{array}{l}\text { Biotic (Fungi, bacteria, virus, } \\
\text { nematodes), drought, cold, } \\
\text { salinity, heat } \downarrow ; \quad \text { Xanthomonas } \\
\text { oryzae \& drought } \uparrow\end{array}$ & $\begin{array}{l}\text { decreased } \\
\text { metabolic rate }\end{array}$ & $\begin{array}{l}\text { Mantri et al. 2010, } \\
\text { Prasch \& Sonnewald } \\
\text { 2013; Shaik \& } \\
\text { Ramakrishna 2013; } \\
\text { Atkinson 2011; Zhang et } \\
\text { al. 2015; Narsai et al. } \\
2013\end{array}$ \\
\hline Cytochrome p450 & A. Thaliana & $\begin{array}{l}\text { Nematode } \& \text { drought } \downarrow ; \text { Bacteria, } \\
\text { fungi, drought, salt } \& \text { heat } \uparrow \& \downarrow\end{array}$ & $\begin{array}{l}\text { cell wall } \\
\text { reinforcement }\end{array}$ & $\begin{array}{l}\text { Atkinson 2011; Narsai et } \\
\text { al. 2013, Shaik \& } \\
\text { Ramakrishna 2013; }\end{array}$ \\
\hline Peroxidase & $\begin{array}{l}\text { A. Thaliana, } O . \\
\text { sativa }\end{array}$ & Biotic, cold, drought $\downarrow$ & $\begin{array}{l}\text { generating } \\
\mathrm{H}_{2} \mathrm{O}_{2}\end{array}$ & $\begin{array}{l}\text { Narsai et al. 2013; Shaik } \\
\text { \& Ramakrishna 2013; }\end{array}$ \\
\hline
\end{tabular}

Abbreviations: nd: not determined; $\uparrow:$ up-regulated; $\downarrow$ : down-regulated; $\uparrow \& \downarrow$ : some members were up-regulated, some were down-regulated.

expressed among the fungal pathogen and one or more of the abiotic stresses, while no transcript was commonly regulated across all the four stresses assessed.

In $A$. thaliana, Sham et al. using microarray mRNA expression profiling found that the genes regulated in response to Botrytis cinerea were more similar to those induced by osmotic stress than genes differentially expressed in response to heat and salt stresses. Conversely, 13 stress-inducible and 29 stress-repressible genes were responsive to all four stresses (Sham et al. 2015). In 2013, in-depth transcriptome analysis of potato (Solanum tuberosum L.) using RNA-Seq was conducted in response to biotic stress, potato late blight fungus (Phytophthora infestans), several abiotic stresses i.e. salinity, drought, and heat, and plant hormone treatment (abscisic acid, 6-benzylaminopurine, gibberellic acid, and indole-3-acetic acid (Massa et al. 2013). Their results exhibited that Pathogenrelated genes are more specifically induced than abiotic- and hormone-related genes and hormones and abiotic stresses share proportionately more differentially expressed genes than each does with the biotic stress. Further, a weighted gene coexpression network analysis revealed that $50 \%$ of the differentially expressed genes were not specific and expressed in response to two or more stress conditions, whilst the $50 \%$ remaining were stressspecific genes that were only regulated in response to single environmental factor (Massa et al. 2013). The results of this study exhibited a degree of overlap in the defence response to pathogen infection and abiotic stresses, but a unique response to each environmental stress.

Further, Weston et al. 2011 using a weighted gene co-expression network approach found that heat shock proteins respond to heat stress in a species- 
specific manner. A gene co-expression network analysis of 1094 microarrays of Arabidopsis using a non-target approach revealed that genes involved in photosynthesis, protein synthesis and response to oxidative stress are also largely involved in response to environmental stresses (Mao et al. 2009).

Another study indicated that the course of day affected transcriptome response of the plant to drought and the response can vary with the time of day (Wilkins et al. 2010). In addition, responses of different plant species to drought is not just affected by the time of day but also clone history and location (Raj et al. 2011). The authors investigated the impact of individual environmental history on response to drought stress and their results showed that differences in transcript abundance patterns in response to drought was based on differences in geographic origin of clones, suggesting the interplay between genotype and environment.

Therefore, the level of crosstalk between different defence response pathways and plant immune responses to single stresses and the combination of different environmental stimuli depend upon several factors such as plant species, tissues, developmental stage, stressors, stress intensity, time series, time of day, geographic origin, etc. (Wu et al. 2009; Mantri et al. 2010; Skibbe et al. 2010; Cramer et al. 2011; Weston et al. 2011; Massa et al. 2013; Shaik et al. 2013; Asai and Shirasu 2015; Li et al. 2015; Pandey et al. 2015). Further, these data demonstrate the complex nature of interactions and exhibit that the complex mechanisms comprising overlap and specific signaling pathways fine-tune plant responses.

Collectively, multi-parallel single stress along with the combination of single stresses require to be investigated at spatial and temporal resolution, as comparative spatiotemporal gene expression analysis, to identify a unique response modulated by combined stress. Here, we discuss the recent advances in the understanding of the mechanisms underlying plant immunity, at the molecular level, against biotic and abiotic stresses and the latest findings on the signaling crosstalk in response to the combination of biotic and abiotic stresses.

\section{Receptor proteins as frontline defence}

Plant pattern-recognition receptors (PRRs) as a frontline of the immune system are an extremely diverse family of cell surface-localized receptors. They are synthesized in the endoplasmic reticulum and transported to the plasma membrane via the secretory pathway (Frescatada-Rosa et al. 2015). PRRs have been classified into receptor-like kinases (RLKs) and receptor-like proteins (RLPs) containing extracellular ligand-binding domains that perceive a wide range of extracellular signals such as PAMPs and abiotic stresses. They trigger the downstream intracellular stress signaling cascades by phosphorylation of intracellular serine/threonine kinase domains (Osakabe et al. 2013; Zipfel 2014; Trdá et al. 2015). RLKs form one of the largest gene superfamily in the genomes of different plant species with 610 and 1131 members in Arabidopsis and rice, respectively (Gish and Clark 2011). They are classified into 44 subfamilies including lysine motifs, lectin, epidermal growth factor-like repeats, self-compatibility domain (S-domain), wallassociated kinase (WAK) and leucine-rich repeats (LRRs) (Frescatada-Rosa et al. 2015; Trdá et al. 2015). The latter constitute the largest group of RLKs in plants which play essential roles in a wide range of processes governing growth and development as key regulators, grain yield components improvement, hormone perception, initiating innate immune defence at front-line against microbial pathogens and adaptation to abiotic stresses (Osakabe et al. 2013; Macho and Zipfel 2014; Zou et al. 2015).

The role of LRR-RLKs in pathogen sensing and activation of downstream defence responses has been reviewed in depth lately (Macho and Zipfel 2014; Frescatada-Rosa et al. 2015; Trdá et al. 2015). Despite, there is growing evidence indicating that RLKs can have positive and negative regulatory role in both biotic and abiotic stress response (Tanaka et al. 2012; Zhao et al. 2013; Jun et al. 2015).

Recent studies revealed that $G b R L K$, a receptorlike kinase (RLK) gene, involved in response to both biotic and abiotic stresses is a positive regulator. GbRLK increased salinity and drought stress tolerance through reduction of water loss rate in leaves and increased sensitivity to $A B A$ and enhanced tolerance to Verticillium wilt in GbRLK transgenic cotton and Arabidopsis lines (Zhao et al. 2013; Jun et al. 2015). In recent past, Jun et al. used transgenic GbRLK cotton and Arabid-opsis lines to reveal that overexpression of GbRLK modulated expression of several genes involved in biotic and abiotic stresses and suggested that the increased resistance to Verticillium dahliae infection in transgenic plants could be a result of reduction in the water damage losses and 
regulation of defence-related gene expression (Jun et al. 2015). These findings suggest that GbRLK could possibly be a key regulator of both, biotic and abiotic signaling.

In another study, $A B A$ and osmotic stress signal transduction in $A$. thaliana is negatively regulated by a receptor-like cytosolic kinase (RLCK) gene, $A R C K 1$, that encodes a cytosolic protein kinase belonging to the RLCK family and cysteine-rich repeat (CRR) RLK sub-family (Tanaka et al. 2012). The arck1 knockout mutant showed higher sensitivity to ABA and osmotic stress than the wildtype. Microarray analysis using CRK36 RNAi transgenic plants exhibited significantly increased ABA-responsive gene expression. CRK36 is another member of the CRR RLK sub-family that interacts with $A R C K 1$ to form a complex to fine-tune defence responses against abiotic stresses and adjust growth via hormone signaling according to the growth phase. Hence, ARCK1 is not involved in the suppression of ABA-responsive genes per se (Tanaka et al. 2012).

As discussed, RLKs are multifunctional receptors which are involved in regulation of plant responses to both, biotic and abiotic stresses. Altogether this suggests a conserved mechanism for signal perception and activating downstream signaling networks, hence PRRs could be signal recognition receptors (SRRs). However, a mechanism for crosstalk of signal perception in response to environmental stimuli remains to be addressed.

\section{Transcription factors}

Transcription factors (TFs) include members of the APETALA2/ETHYLENE-RESPONSE ELEMENT BINDING FACTOR (AP2/ERF), basic-helix-loophelix (bHLH), basic domain leucine zipper (bZIP), MYB, NAC and WRKY families (Ford et al. 2015). TFs are the central core of the gene regulatory networks and precisely modulate the transcription rate through either repression or activation of gene expression. They are multi-functional and mediate diverse aspects of developmental processes and responses to various biotic and abiotic stimuli in plants (Tsuda and Somssich 2015).

Carrera et al. 2009 performed a gene co-expression network analysis of 1,436 microarray experiments of Arabidopsis using InferGene software. Their results showed that several TFs were the most central regulatory hubs including KAN3 involved in auxin, MYB29 in gibberellin, MYB121 in abscisic acid, and ERF1 in ethylene responses.
The Arabidopsis BOTRYTIS SUSCEPTIBLE 1 (BOS1) gene encoding an R2R3MYB transcription factor conferred resistance to pathogens and tolerance to salt and drought abiotic stress factors through a JA-mediated defence signaling pathway. BOS1-deficient mutant bos1 displayed increased susceptibility to necrotrophic fungi $B$. cinerea and Alternaria brassicicola and biotrophic bacterial pathogen Pseudomonas syringae and impaired tolerance to abiotic stresses salinity, drought and oxidative stress (Mengiste et al. 2003). The results of this study indicate that MYB TFs play essential roles in multiple stress responses.

Functional studies of the Arabidopsis Botrytis Susceptible1 Interactor $(B O /)$ and three $\mathrm{BOI}$ RELATED GENES (BRGs) encoding a subclass of RING E3 ligases have revealed that $B O I$ interacts with BOS1 and ubiquitinates it to confine the extent of cell death induced by pathogens and abiotic stresses (Luo et al 2010). B. cinerea and salt induced the expression of $\mathrm{BOI}$ but its expression was intriguingly suppressed by gibberellins. $\mathrm{BO} /$ RNA interference (RNAi) plants showed more susceptibility to fungal pathogen and salt stress and reduced growth responsiveness to gibberellin. Hence, $B O I$ and $B R G s$ are associated with resistance to both, biotic and abiotic stresses via suppression of cell death which is different from HR cell death mediated by the disease resistance genes RPM1 and RPS2 (Luo et al 2010). This finding suggests that the expression of $B O /$ gene is complex and regulated by multiple factors and there is hormonal crosstalk between JA and GA to regulate the expression of BOS1. Further, GA may act as a negative regulator of $\mathrm{BOI}$. However, more comprehensive analysis is required to dissect the complex interacting pathways with cross-talk at different levels.

A transcription factor of the NAC family, one of the largest TFs family that is only found in plants, Arabidopsis thaliana activating factor1 (ATAF1) is induced in response to various biotic and abiotic stresses. ATAF1 is drought-inducible gene and it was significantly expressed in an ABA-deficient mutant aba2 in response to drought and high salinity, demonstrating that ATAF1 expression is ABA-independent. ATAF1 is also involved in stomatal regulation and extensively expressed in stomatal guard cells (Lu et al. 2007; Wu et al. 2009). Therefore, ATAF1 may augment plant drought tolerance by stomatal closure via both ABAdependent and $A B A$-independent signaling pathway. 
On the other hand, ABA biosynthesis aldehyde oxidase gene (AAO3) was induced in ataf1 mutant plants upon biotrophic powdery mildew fungal pathogen Blumeria graminis f.sp. hordei (Bgh) infection and compromised penetration resistance towards Bgh (Jansen et al. 2008). By contrast, ABA biosynthesis-deficient mutant was highly resistant to attempted Bgh penetration. These data suggest that ATAF1 is a stimulus-dependent transcriptional regulator of $A B A$ biosynthesis and $A B A$ is a negative regulator of penetration resistance. Moreover, the expression of JA/ET-activated transcription of plant defensin genes was suppressed in ataf1 mutants whereas induced in the wild-type plants (Jansen et al. 2008). Further, barley ATAF1 homologue HvNAC6 is also a positive regulator of basal defence and offers penetration resistance towards virulent Bgh attack (Jensen et al. 2007) (Figure 3).

Collectively, ATAF1 as a point of crosstalk, play a critical role in the regulation of antagonistic interplay between $\mathrm{ABA}$ - and JA/ET-mediated signaling and regulates the inhibitory effect of $A B A$ on JA/ETactivated transcription of plant defensin genes to enhance plant tolerance in response to biotrophic fungus.
Moreover, ATAF1 knockout mutant positively regulates the expression of several stressresponsive genes such as COR47, ERD10, KIN1, $R D 22$ and $R D 29 A$ under drought stress (Lu et al. 2007; Wu et al. 2009). By contrast, overexpression of ATAF1 in transgenic plants ATAF1-OE increased susceptibility to $B$. cinerea, Alternaria brassicicola, and $P$. syringae pv. Tomato, whilst ATAF1 chimeric repressor construct enhanced disease resistance to these pathogens via up-regulation of pathogenesisrelated (PR) genes, PR-1 and PR-5, and JA/ETmediated defence responsive gene plant defensin1.2 (PDF1.2) (Lu et al. 2007). In line with this, Prasch and Sonnewald found that the expression of $P R$ genes and $R$ genes-mediated disease resistance was suppressed and virus resistance was compromised in virus infected $A$. thaliana under simultaneous double drought and heat stress, whereas PR1,PR2, and PR5 were induced in response to single virus infection (Prasch and Sonnewald, 2013).

Therefore, these studies corroborate that ATAF1 is a repressor of basal defence and negative regulator of disease resistance, which seems to be ABAmediated, in response to both, necrotrophic fungi

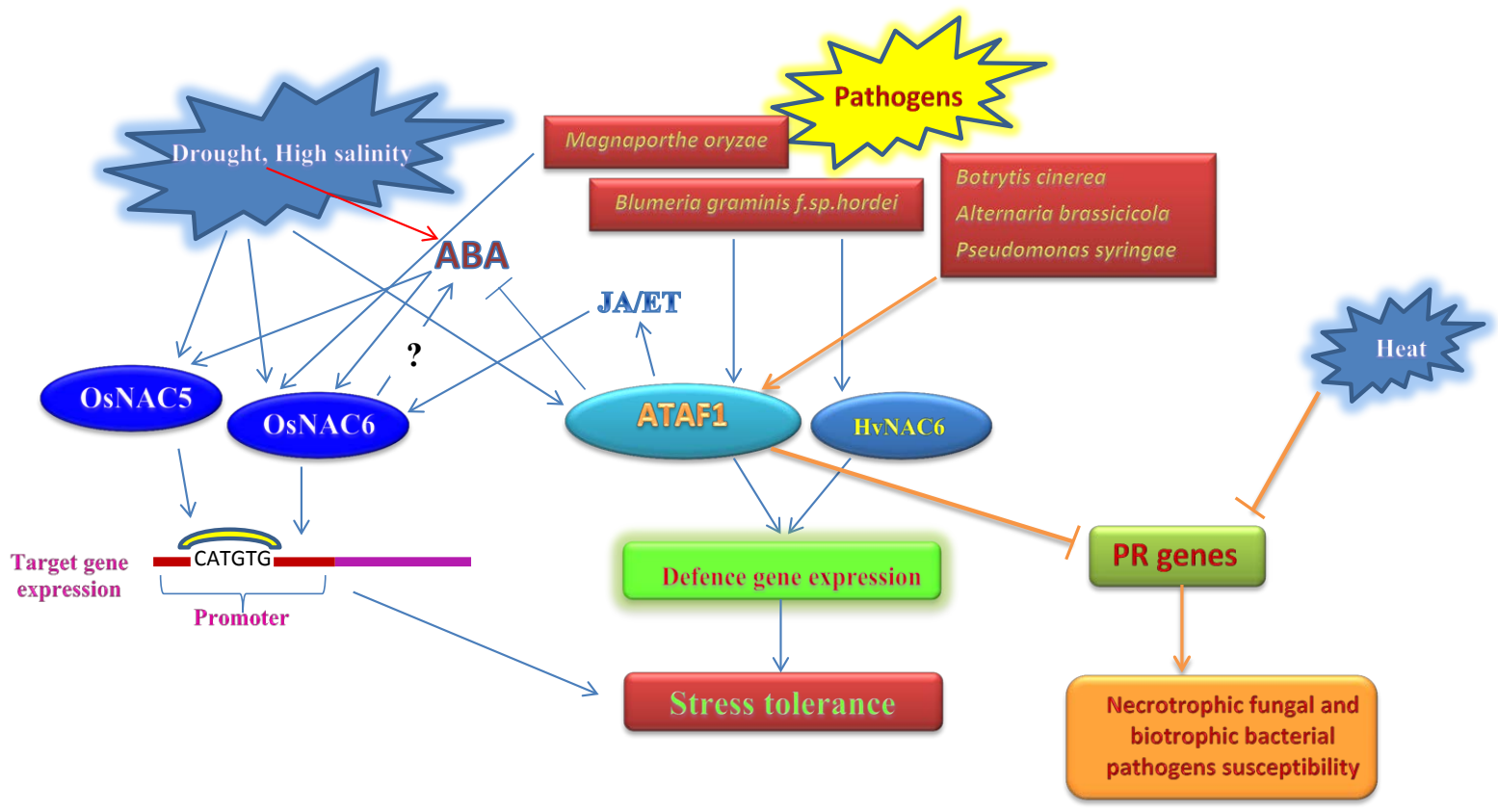

Figure 3. A schematic representation of transcriptional regulatory networks of NAC transcription factors in biotic and abiotic stresses responses. 
and biotrophic bacterial pathogen through repression of PRs (Wang et al. 2009).

Recently, a novel role has been identified for ATAF1 as a positive regulator of senescence via activating senescence-promoting TF ORESARA1 (AtNAC092) and repressing MYB transcription factor GOLDEN2LIKE1 (GLK1) by directly binding to their promoters (Garapati et al. 2015). These findings demonstrate that ATAF1 is a multifaceted TF with versatile capabilities and plays an important role in mediating crosstalk between biotic and abiotic stress responses, though further research is required to unravel the role of senescence-associated genes in defence response and the correlation between ATAF1, hormone signaling, and disease resistance.

The OsNAC6 gene, another member of the NAC family which has high similarity to genes in the ATAF subfamily is a positive regulator of defence in response to both, biotic and abiotic stresses (Nakashima et al. 2007). OsNAC6 was induced by cold, drought, high salinity, wounding, ABA, JA, and blast disease in rice (Ohnishi et al. 2005; Nakashima et al. 2007; Takasaki et al. 2010). Transgenic rice plants over-expressing OsNAC6 exhibited enhanced tolerance to drought and high salinity and some tolerance to hemibiotrophic fungal pathogen Magnaporthe oryzae. Further, microarray analysis revealed that many stress-responsive genes were up-regulated in OsNAC6overexpressing rice plants, though OsNAC6 has a negative effect on growth and productivity in rice (Nakashima et al. 2007) (Figure 3). Interestingly, OsNAC5 homolog of OsNAC6 was induced by drought, cold, high-salinity, abscisic acid and methyl jasmonic acid and augmented stress tolerance by overexpression of stress-responsive genes such as OsLEA3 with no negative effect on rice growth (Takasaki et al. 2010). Notwithstanding, the crosstalk between OsNAC6/OsNAC5 and hormone signaling underlying tolerance to multiple biotic and abiotic stresses requires further investigation.

\section{Heat shock proteins and heat shock trans- cription factors}

A family of highly conserved genes encodes heat shock proteins (HSPs) across all cells of both, prokaryotes and eukaryotes. These stress response proteins are constitutively expressed in cells under normal conditions, in the absence of environmental stresses, and function as molecular chaperones regulating protein synthesis, folding, assembly, translocation, and degradation (Wang et al. 2004). Their expression is increased upon exposure to a wide variety of environmental stimuli to protect cells against stress by preventing protein aggregation and stabilizing misfolded proteins and cellular homeostasis (Wang et al. 2004). Recent findings reveal that over-expression of OsHSP18.6 augmented tolerance to diverse types of abiotic stresses including heat, drought, salt and cold in rice (Wang et al. 2015). In addition, genes encoding HSPs were induced in Arabidopsis by necrotrophic fungus Botrytis cinerea infection, cold, drought and oxidative stress (Sham et al. 2014). Therefore, HSPs may regulate pathogen defence as well as abiotic stress tolerance.

The transcription of HSPs is primarily regulated by heat shock transcription factors (HSFs) which bind to highly conserved motifs of the promoter regions of HSP genes known as heat stress-elements (HSEs; 5' -AGAAnnTTCT-3') resulting in increased tolerance to biotic and abiotic stresses ( $\mathrm{Hu}$ et al. 2015; Virdi et al. 2015). Recently, genome-wide transcriptional analyses in a number of plants have shown that HSFs have an integral role in response to biotic and abiotic stresses such as heat, cold, salt, drought and oxidative stress (Chung et al. 2013; Xue et al. 2015). For instance, several HSF genes were significantly induced in strawberry Fragaria vesca by heat, drought, cold, and salt stresses and by biotic stress powdery mildew infection, and biotrophic fungus Podosphaera aphanis (Hu et al. 2015). Further, the level of AtHsfA6a transcript highly increased under high salinity and dehydration conditions (Hwang et al. 2014). In addition, HSF4 was induced by salinity and/or osmotic stress and fungal pathogen Botrytis cinerea in A. thaliana (Sham et al. 2015) while HsfA3 was reported to be associated with response to drought and salt stress (Li et al. 2013). Moreover, several Hsf genes were highly over-expressed by exogenous ABA (Hwang et al. 2014; Hu et al. 2015). These studies thus provide a link between ABA and Hsfs activation and indicate that HSFs, as a point of cross-tolerance, act as the transcriptional activators of several stress-responsive genes which could be via ABA-dependent signaling pathway. They also suggest the involvement of HSFs-activated stressresponsive genes in HSFs-mediated stress tolerance.

Recent studies identified melatonin ( $\mathrm{N}$-acetyl-5methoxytryptamine) as an important secondary messenger that conferred resistance to heat, salt, drought, cold and pathogen Pseudomonas syringe pv. tomato in Arabidopsis (Shi et al. 2015 a,b). It positively regulated the expression of class $A 1$ heat- 
shock factors (HSFA1s) as the master regulators of heat stress response and several stress-responsive genes such as COR15A, RD22, and KIN1. Hence, melatonin may remarkably impact crosstalk among stress responses, and control biotic and abiotic stress response as a regulator of HSFs (Shi et al. $2015 a, b)$.

There is also evidence to suggest a crosstalk between HSFs and oxidative stress signaling, and HSPs and TIR-NBS-LRR genes (Scarpeci et al. 2008; Prasch and Sonnewald 2013). Scarpeci et al. identified heat shock transcription factors HsfA2 and HsfA4A that can sense oxidative stress caused during heat and pathogen attack via direct sensing of $\mathrm{H}_{2} \mathrm{O}_{2}$ in Arabidopsis (Scarpeci et al. 2008). Prasch and Sonnewald investigated the response of Arabidopsis plants to heat, drought, and Turnip mosaic virus (TuMV) by transcriptome and metabolome analysis. Their results revealed that the highest number of TIR-NBS-LRR resistance genes is induced under heat and combined heat and drought stress, whilst only a few TIR-NBS-LRR genes were regulated under combined triple stress. These findings suggest that HSPs regulate the function of NBS-LRR genes in response to biotic and abiotic stresses (Prasch and Sonnewald 2013).

\section{MicroRNAs}

MicroRNAs (miRNAs) are a class of endogenous small non-coding, single-stranded RNAs of approximately 20-24 nucleotides in length. They regulate expression of their target genes in plants as key post-transcriptional gene regulators through translational repression or transcript cleavage (Mantri et al. 2013; Kamthan et al. 2015). miRNAs have been implicated in plant growth and development, cell proliferation and cell death, organogenesis, auxin signaling and also established to perform key roles in response to a wide array of biotic and abiotic stresses. (Khraiwesh et al. 2012; Mantri et al. 2013). miRNAs target transcription factors comprising AP2, bHLH, MYB, TCP, NAC, NFY, and HD-Zip and diverse range of defencerelated genes such as receptor-like kinase, MAPK genes, peroxidase, NB-LRRs, and ABC transporters (Kulcheski et al. 2011; Khraiwesh et al. 2012; Kumar 2014; Raghuram et al., 2014; Omidvar et al. 2015). Recent studies revealed that miRNAs respond to abiotic stresses in a genotype-, tissue-, stress-, and miRNA-dependent manner (Omidvar et al. 2015; Zhang 2015). Negative correlation, however, has prevailed between the expression levels of miRNAs implicated in the response to biotic and abiotic stresses and their target genes (Xin et al. 2010; Raghuram et al., 2014; Omidvar et al. 2015).

Only a few studies have been conducted on the role of miRNA in plant responses to both, biotic and abiotic stresses. Using Solexa high-throughput sequencing, Xin et al. define the role of miRNAs in regulating the response of wheat to heat stress and powdery mildew infection caused by Erysiphe graminis f.sp. tritici (Egt). They identified 24 and 12 miRNAs that were expressed with different expression patterns in response to powdery mildew infection and heat stress, respectively. Further, 9 miRNAs were co-regulated by both, powdery mildew and heat, of which miR827 and miR2005 were overexpressed in both, Egt and heat stress. This exhibits their important role in wheat response to biotic and abiotic stresses. Further, miR156 was down-regulated in Egt susceptible (Jindong8) and resistant (Jindong8-Pm30) wheat lines after Egt inoculation whilst it was up-regulated in both, heat susceptible (Chinese Spring) and tolerant (TAM107) genotypes, after heat treatment. In addition, negative correlations were identified between miR156 and its target genes Ta3711 and Ta7012 (Xin et al. 2010). These studies reveal cross-talk and functional interactions among stress-induced wheat miRNAs and corroborate the stress-specific role of miRNAs.

Kulcheski et al. (2011) using Solexa sequencing technology analyzsed the miRNA expression pattern of soybean cultivars susceptible and resistant to Asian soybean rust, Phakopsora pachyrhizi, to pathogen infection and drought stress. Their results revealed that many miRNAs are involved in response to both, biotic and abiotic stresses, though they exhibited completely different and contrasting expression profiles in the response to rust infection and drought stress. In addition, some miRNAs respond to the same stress differently depending on the soybean genotypes. Of these, MIR-Seq11 predicted to target peroxidase precursor mRNAs, was significantly induced in the roots of drought susceptible genotype (BR 16) compared to the controls, although it responds similarly in the treated and untreated drought-tolerant cultivar (Embrapa 48). Further, expression of MIR-Seq11 was remarkably different between the mock and rust inoculated susceptible genotype (Embrapa 48), while it responds in a similar manner in the soybean rust resistant genotype (PI561356) (Kulcheski et al. 2011). These findings suggest that miRNAs may respond to stresses in a not only stress- but also genotype-specific manner. 
The results also indicate that miRNAs may target only one locus or several loci in response to biotic and abiotic stresses. MIR-Seq10, MIR-Seq15, and MIR-Seq18 targeted only one locus, although, MIRSeq05, MIR-Seq11, MIR-Seq16 and MIR-Seq19 presented several loci as targets (Kulcheski et al. 2011).

Recently, Omidvar et al. (2015) used Illumina Hiseq2000 platform to investigate the simultaneous impact of $A B A$ and mannitol treatments on the wildtype and abiotic stress-tolerant $7 B-1$ tomato mutant. They also analyzsed the expression of 6 selected miRNAs (including miR159, miR166, miR472, miR482, miR\#A, and miR\#D) in response to ABA, mannitol, $\mathrm{NaCl}$ and cold treatments. Their results revealed that miRNAs were differentially expressed in response to abiotic stresses. miRNA159 and miRNA166 were inversely expressed in response to cold, while they showed the same pattern in response to $\mathrm{ABA}$, mannitol, and $\mathrm{NaCl}$. Likewise, different levels of expression were revealed between hypocotyl and root tissues. miR159, miR472, and miR482 were remarkably downregulated in roots compared with hypocotyl. These results support previous studies that found miRNAs react to abiotic stresses in a stress-, miRNA-specific and plant tissue-dependent manner.

While there is currently no published study on miRNA responses to combined stresses and crosstalk between miRNAs in response to a combination of stresses, it is indispensable to dissect their responses, functions, and regulatory mechanisms in response to the combination of biotic and abiotic stresses.

\section{Epigenetics and stress response}

The term epigenetics describes heritable changes in gene expression patterns that transpire without altering the underlying DNA sequence (Iwasaki and Paszkowski 2014). In the recent past, numerous studies have corroborated the crucial role of epigenetic mechanisms in plant immunity and stress adaptation (Ding and Wang 2015; Iwasaki and Paszkowski 2014; Kim et al. 2015; Kinoshita and Seki 2014) and ample progress has been made in elaborating epigenetic regulation of plant responses to stressors (Álvarez-Venegas and De-la-Peña 2015; Ding and Wang 2015; Dowen et al. 2012; Su et al. 2015). The results of these studies demonstrated that plants have evolved intricate epigenetic mechanisms in regulating plant responses to environmental factors comprising DNA methylation, histone posttranslational modification, chromatin remodeling complexes, small non-coding RNAs (miRNAs and small interfering RNAs, siRNAs), and long non-coding RNAs.

DNA methylomes profiling of Arabidopsis thaliana plants challenged with a bacterial pathogen, Pseudomonas syringae pv. tomato, nonpathogenic bacteria or salicylic acid (SA) revealed that stressinduced differentially methylated regions (DMRs) can regulate the expression of proximal stressresponsive genes as general regulatory hubs for defence-responsive genes. Moreover, their results exhibited that SA-induced demethylation, along with up-regulation of transposable elements and 21-nt small RNAs produced from these elements increased the expression of neighboring proteincoding genes in SA-treated plants (Dowen et al. 2012).

The results of another study indicate the involvement of AhHDA1, an RPD3/HDA1-like superfamily histone deacetylase (HDAC), in the regulation of stress resistance genes in response to osmotic stress and ABA in peanut (Arachis hypogaea) (Su et al. 2015).

The review by Liu et al (2015) describe the major epigenetic mechanisms contributing to the control of plant heat responses, highlighting epigenetic modifications comprising DNA methylation, histone modifications, histone chaperones, histone variants, ATP-dependent chromatin remodeling, small noncoding RNAs and long non-coding RNAs in modulating the expression of heat-responsive genes under high temperature. In another review, Kim et al (2015) highlight the role of histone modification in stress memory and modulating stress-responsive gene networks in response to drought, salinity, heat, and cold.

Further, another finding unravelled that when plants confront stressful environmental conditions, the first experience of stress can be memorized and inherited to the next generation as a transgenerational epigenetic inheritance (Molinier et al. 2006). Recent studies revealed epigenetic mechanisms underlying this stress-driven phenomenon, although are not fully understood. The evidence suggests full-scale reprogramming of the epigenome does not occur during gametogenesis due to escaping reprogramming of epigenetic marks (Bilichak and Kovalchuk 2016). 
Nevertheless, stress-responsive epigenomes have thus far been studied in response to single biotic/or abiotic stresses. Studies of the epigenome, however, are scarce in simultaneous biotic and abiotic stresses. Extending our knowledge of epigenetic mechanisms to simultaneous combined stresses is not only essential for understanding the crosstalk between epigenetic components, and epigenome and transcriptome but to also providing vital information for epigenetic engineering and crop improvement. Though, we are still far from being able to incorporate it in plant breeding for crop improvement.

\section{Conclusions and perspectives}

Plants have evolved complex mechanisms enabling them as sessile organisms to survive not only against single stresses but also against a combination of stresses. Understanding complexity of plant defense mechanisms and stress signaling is indispensable in order to minimize the impact of stresses on plant growth, development, and productivity and maximize their lifespan. In the recent past, studies on plant immune responses to individual and combination of biotic and abiotic stress have revealed the complex nature and high degree of signaling crosstalk in responses to various attackers. Nevertheless, understanding signaling crosstalk between components of the plant defence pathways under combined stress conditions is still limited, although it is continually evolving due to remarkable advances in highthroughput sequencing technologies and powerful bioinformatics tools for genome-wide study, transcriptome, and proteome analysis. Recent studies demonstrate that production of ROS, MAPkinase cascades, and hormone signaling are triggered by both, biotic and abiotic environmental stimuli in a non-specific manner and play crucial roles in the response to biotic and abiotic stresses as common components of the immune system. Receptor proteins as a frontline of the immune system are key regulators of crosstalk between biotic and abiotic signaling. Transcription factors as positive and negative regulators of plant defence also play a significant complex role in crosstalk and fine-tuning responses to combined biotic and abiotic stresses. Though, interactions between TFs involved in the regulation of stress- and hormoneresponsive genes are still needed to be elucidated. Further, identification of both, common and specific components of the signaling crosstalk in plants, will allow development of novel tools to combat the combination of biotic and abiotic stresses usually encountered in the field conditions. Hence, identification of multifunctional genes and genes responsible for biotic and abiotic stress resistance would help accelerate development of resistant crop plants through precise genome editing using emerging technologies for targeted genome editing such as Transcription Activator-Like Effector Nucleases (TALENs) and CRISPR (Clustered Regulatory Interspaced Short Palin-dromic Repeats)/Cas9 systems.

\section{Declaration of interest}

The authors report no declarations of interest.

\section{References}

Álvarez-Venegas, R., De-la-Peña, C. (2015). Editorial: recent advances of epigenetics in crop biotechnology. Front. Plant Sci. 7, 413.

Asai, S., and Shirasu, K. (2015). Plant cells under siege: plant immune system versus pathogen effectors. Curr. Opin. Plant Biol. 28, 1-8.

Atkinson, N.J., Lilley, C.J., and Urwin, P.E. (2013). Identification of genes involved in the response of Arabidopsis to simultaneous biotic and abiotic stresses. Plant Physiol. 162, 2028-2041.

Atkinson N.J., and Urwin P.E. (2012). The interaction of plant biotic and abiotic stresses: from genes to the field. J. Exp. Bot. 63, 3523-3544.

Atkinson, N.J. (2011). Plant molecular response to combined drought and nematode stress. Ph.D. thesis, University of Leeds. http:// etheses.whiterose.ac.uk/2131/

Bilichak, A., Kovalchuk, I. (2016). Transgenerational response to stress in plants and its application for breeding. J. Exp. Bot. 67, 2081-92.

Carrera, J., Rodrigo, G., Jaramillo, A., and Elena, S.F. (2009). Reverse-engineering the Arabidopsis thaliana transcriptional network under changing environmental conditions. Genome Biol. 10, R96.

Chung, E., Kim, K.M., and Lee, J.H. (2013). Genome-wide analysis and molecular characterization of the heat shock transcription factor family in Glycine max. J. Genet. Genomics 40, 127-135.

Cramer, G.R., Urano, K., Delrot, S., Pezzotti, M., and Shinozaki, K. (2011). Effects of abiotic stress on plants: a systems biology perspective. BMC Plant Biol. 11, 163.

Cui, H., Tsuda, K., and Parker, J.E. (2015). Effectortriggered immunity: from pathogen perception to a robust defense. Annu. Rev. Plant Biol. 66, 487-511.

Ding, B., Wang, G.L. (2015). Chromatin versus pathogens: the function of epigenetics in plant immunity. Front. Plant Sci. 6, 675. 
Dodds, P.N., and Rathjen, J.P. (2010). Plant immunity: towards an integrated view of plantpathogen interactions. Nature Rev. Genet. 11, 539-548.

Dowen, R.H., Pelizzola, M., Schmitz, R.J., Lister, R., Dowen, J.M., Nery, J.R., Dixon, J.E., Ecker, J.R. (2012). Widespread dynamic DNA methylation in response to biotic stress. Proc. Natl. Acad. Sci. USA 109, E2183-91.

Frescatada-Rosa, M., Robatzek, S., and Kuhn, H. (2015), Should I stay or should I go? Traffic control for plant pattern recognition receptors. Curr. Opin. Plant Biol. 28, 23-29.

Fujita, M., Fujita, Y., Noutoshi, Y., Takahashi, F., Narusaka, Y., Yamaguchi-Shinozaki, K., and Shinozaki, K. (2006). Crosstalk between abiotic and biotic stress responses: a current view from the points of convergence in the stress signaling networks. Curr. Opin. Plant Biol. 9, 436-442.

Garapati, P., Xue, G.P., Munné-Bosch, S., and Balazadeh, S. (2015). Transcription factor ATAF1 in Arabidopsis promotes senescence by direct regulation of key chloroplast maintenance and senescence transcriptional cascades. Plant Physiol. 168, 1122-39.

Gish, L.A., and Clark, S.E. (2011). The RLK/Pelle family of kinases. Plant J. 66, 117-127.

Hu, Y., Han, Y.T., Wei, W., Li, Y.J., Zhang, K., Gao, Y.R., Zhao, F.L., and Feng, J.Y. (2015). Identification, isolation, and expression analysis of heat shock transcription factors in the diploid woodland strawberry Fragaria vesca. Front. Plant Sci. 6, 736.

Hwang, S.M., Kim, D.W., Woo, M.S., Jeong, H.S., Son, Y.S., Akhter, S., Choi, G.J., and Bahk, J.D. (2014). Functional characterization of Arabidopsis HsfA6a as a heat-shock transcription factor under high salinity and dehydration conditions. Plant Cell Environ. 37, 1202-1222.

Iwasaki, M., Paszkowski, J. (2014). Epigenetic Memory in plants. EMBO J. 33, 1987-1998.

Jansen, M.K., Hangedorn, P.H., De Torres-Zabala, M., Grant, M.R., Rung, J.H., Colling, D., and Lyngkjaer, M.F. (2008). Transcriptional regulation by an NAC (NAM-ATAF1,2-CUC2) transcription factor attenuates $A B A$ signalling for efficient basal defence towards Blumeria graminis f. $\mathrm{sp}$. hordei in Arabidopsis. Plant J. 56, 867-880.

Jensen, M.K., Rung, J.H., Gregersen, P.L., Gjetting, T., Fuglsang, A.T., Hansen, M., Joehnk, N., Lyngkjaer, M.F., and Collinge, D.B. (2007). The HvNAC6 transcription factor: a positive regulator of penetration resistance in barley and Arabidopsis. Plant Mol. Biol. 65, 137-50.
Jun, Z., Zhang, Z., Gao, Y., Zhou, L., Fang, L., Chen, X., Ning, Z., Chen, T., Guo, W., and Zhang T. (2015). Overexpression of GbRLK, a putative receptor-like kinase gene, improved cotton tolerance to Verticillium wilt. Sci. Rep. 5, 15048.

Kamthan, A., Chaudhuri, A., Kamthan, M., and Datta, A. (2015), Small RNAs in plants: recent development and application for crop improvement. Front. Plant Sci. 6, 208.

Khraiwesh, B., Zhu, J-K., and Zhu, J. (2012). Role of miRNAs and siRNAs in biotic and abiotic stress responses of plants. Biochim. Biophys. Acta 1819,137-148.

Kim, J.M., Sasaki, T., Ueda, M., Sako, K., Seki, M. (2015). Chromatin changes in response to drought, salinity, heat, and cold stresses in plants. Front. Plant Sci. 6, 114.

Kim, Y., Tsuda, K., Igarashi, D., Hillmer, R.A., Sakakibara, H., Myers, C.L., and Katagiri, F. (2014). Signaling mechanisms underlying the robustness and tunability of the plant immune network. Cell Host Microbe. 15, 84-94.

Kinoshita, T., Seki, M. (2014). Epigenetic memory for stress response and adaptation in plants. Plant Cell Physiol. 55, 1859-1863.

Kissoudis, C., Chowdhury, R., van Heusden, S., van de Wiel, C., Finkers, R., Visser, R.G.F., Bai, Y., and van der Linden, G. (2015). Combined biotic and abiotic stress resistance in tomato. Euphytica. 202, 317-332.

Kissoudis, C., van de Wiel, C., Visser, R.G.F., and van der Linden, G. (2014). Enhancing crop resilience to combined abiotic and biotic stress through the dissection of physiological and molecular crosstalk. Front. Plant Sci. 5, 207.

Kreps, J.A., Wu, Y., Chang, H.S., Zhu, T., Wang, X., and Harper, J.F. (2002). Transcriptome changes for Arabidopsis in response to salt, osmotic, and cold stress. Plant Physiol. 130, 2129-2141.

Kulcheski, F.R., de Oliveira, L.F., Molina, L.G., Almerão, M.P., Rodrigues, F.A., Marcolino, J., Barbosa, J.F., Stolf-Moreira, R., Nepomuceno, A.L., Marcelino-Guimarães, F.C., Abdelnoor, R.V., Nascimento, L.C., Carazzolle, M.F., Pereira , G.A.G., and Margis, R. (2011). Identification of novel soybean microRNAs involved in abiotic and biotic stresses. BMC Genom. 12, 307.

Kumar, R. (2014). Role of microRNAs in biotic and abiotic stress responses in crop plants. Appl. Biochem. Biotechnol. 174, 93-115.

Li, Y., Varala, K., and Coruzzi, M. (2015). From milliseconds to lifetimes; tracking the dynamic behavior of transcription factors in gene networks. Trends Genet. 31, 509-515. 
Li, Z., Zhang, L., Wang, A., Xu, X., and Li, J. (2013). Ectopic overexpression of SIHsfA3, a heat stress transcription factor from tomato, confers increased thermotolerance and salt hypersensitivity in germination in transgenic Arabidopsis. PLoS ONE 8, e54880.

Liu, J., Feng, L., Li, J., He, Z. (2015). Genetic and epigenetic control of plant heat responses. Front. Plant Sci. 6, 267.

Lu, P.L., Chen, N.Z., An, R., Su, Z., Qi, B.S., Ren, F., Chen, J., and Wang, X.C. (2007). A novel drought-inducible gene, ATAF1, encodes a NAC family protein that negatively regulates the expression of stress-responsive genes in Arabidopsis. Plant Mol. Biol. 63, 289-305.

Luo, H., Laluk, K., lai, Z., Veronesa, P., Song, F., and Mengiste, T. (2010). The Arabidopsis botrytis susceptible interactor defines a subclass of RING E3 ligases that regulate pathogen and stress responses. Plant Physiol. 154, 1766-1782.

Ma, S., and Bohnert, H.J. (2007). Integration of Arabidopsis thaliana stress-related transcript profiles, promoter structures, and cell-specific expression. Genome Biol. 8, R49.

Macho, A.P., and Zipfel, C. (2014). Plant PRRs and the activation of innate immune signaling. Mol. Cell. 54, 263-272.

Mantri, N., Basker, N., Ford, R., Pang, E., and Pardeshi, V. (2013). The role of micro-ribonucleic acids in legumes with a focus on abiotic stress response. Plant Genome 6, 1-14.

Mantri, N.L., Ford, R., Coram, T.E., and Pang, E.C.K. (2010). Evidence of unique and shared responses to major biotic and abiotic stresses in chickpea. Environ. Exp. Bot. 69, 286-292.

Mantri, N., Pang, E.C.K. and Ford, R. (2010) Molecular Biology for Stress Management. In Climate Change and Management of Cool Season Grain Legume Crops (Yadav, S.S., McNeil, D.N., Weeden, N. and Patil, S.S., eds). Heidelberg: Springer, pp. 377-408. (ISBN 978-90-481-3708-4)

Mantri, N., Patade, V. and Pang, E.C.K. (2014) Recent Advances in Rapid and Sensitive Screening For Abiotic Stress. In Improvement of Crops in the Era of Climate Change (Ahmad P, Wani MR, Azooz MM, Tran LSP eds). New York: Springer pp 37-47 (ISBN 978-1-4614-8823-1)

Mao, L., Van, H.J.L., Dash, S., and Dickerson, J.A. (2009). Arabidopsis gene co-expression network and its functional modules. BMC Bioinformatics 10, 346.

Massa, A.N., Childs, K.L., Buell, R. (2013). Abiotic and biotic stress responses in Solanum tuberosum group Phureja DM1-3 516 R44 as measured through whole transcriptome sequencing. Plant Genome 6, 1-10.

Mengiste, T., Chen, X., Salmeron, J.M., and Dietrich, R.A. (2003). The BOS1 gene encodes an R2R3MYB transcription factor protein that is required for biotic and abiotic stress responses in Arabidopsis. Plant Cell 15, 2551-2565.

Molinier, J., Ries, G., Zipfel, C., Hohn, B. (2006). Transgeneration memory of stress in plants. Nature 442, 1046-9.

Nakagami, H., Pitzchke, A., and Hirt, H. (2005). Emerging MAP kinase pathways in plant stress signaling. Trends Plant Sci. 10, 339-346.

Nakashima, K., Tran, L.S., Van Nguyen, D., Fujita, M., Maruyama, K., Todaka, D., Hayashi, N., Shinozaki, K., and Yamaguchi-Shinozaki, K. (2007). Functional analysis of a NAC-type transcription factor OsNAC6 involved in abiotic and biotic stress-responsive gene expression in rice. Plant J. 51, 617-30.

Narsai, R., Wang, C., Chen, J., Wu, J., Shou, H., and Whelan, J. (2013). Antagonistic, overlapping and distinct responses to biotic stress in rice (Oryza sativa) and interactions with abiotic stress. BMC Genomics 14, 93.

Nejat, N., Rookes, J., Mantri, N., and Cahill, D. (2016). Plant-pathogen interactions: toward development of next-generation disease-resistant plants. Crit. Rev. Biotechnol. doi: 10.3109/07388551.2015.1134437.

Ohnishi, T., Sugahara, S., Yamada, T., Kikuchi, K., Yoshiba, H.Y., Hirano, H.Y., and Tsutsumi, N. (2005). OsNAC6, a member of the NAC gene family, is induced by various stresses in rice. Genes Genet. Syst. 80, 135-139.

Omidvar, V., Mohorianu, I., Dalmay, T., and Fellner, M. (2015). MicroRNA regulation of abiotic stress response in $7 B-1$ male-sterile tomato mutant. Plant Genome 8, 1-13.

Osakabe, Y., Yamaguchi-Shinozaki, K., Shinozaki, K., and Phan Tran, L. (2013). Sensing the environment: key roles of membrane-localized kinases in plant perception and response to abiotic stress. J. Exp. Bot. 64, 445-458.

Pandey, P., Ramegowda, V., and Senthil-Kumar, M. (2015). Shared and unique responses of plants to multiple individual stresses and stress combinations: physiological and molecular mechanisms. Front. Plant Sci. 6, 723.

Perez, I.B., and Brown, P. (2014). The role of ROS signaling in cross-tolerance: from model to crop. Front. Plant Sci. 5, 754.

Prasch, C.M., and Sonnewald, U. (2013). Simultaneous application of heat, drought and virus to Arabidopsis plants reveals significant 
shifts in signaling networks. Plant Physiol. 162, 1849-1866.

Raghuram, B., Sheikh, A.H., and Sinha, A.K. (2014). Regulation of MAP kinase signaling cascade by microRNAs in Oryza sativa. Plant Signal Behav. 9, e972130.

Raj, S., Brautigam, K., Hamanishi, E.T., Wilkins, O., Thomas, B.R., Schroeder, W., Mansfield, S.D., Plant, A.L., and Campbell, M.M. (2011). Clone history shapes Populus drought responses. Proc. Natl. Acad. Sci. U.S.A. 108, 12521-12526.

Rasmussen, S., Barah, P., Suarez-Rodriguez, M.C., Bressendorff, S., Friis, P., Costantino, P., Bones, A.M., Nielsen, H.B., and Mundy, J. (2013). Transcriptome responses to combinations of stresses in Arabidopsis. Plant Physiol. 161, 1783-1794.

Scarpeci, T.E., Zanor, M.I., and Valle, E.M. (2008). Investigating the role of plant heat shock proteins during oxidative stress. Plant Signal Behav. 3, 856-857.

Schwessinger, B., Bart, R., Krasileva, K., Coaker, G. (2015). Focus issue on plant immunity: from model systems to crop species. Front. Plant Sci. 6, 195

Seki, M., Narusaka, M., Ishida, J., Nanjo, T., Fujita, M., Oono, Y., Kamiya, A., Nakajima, M., Enju, A., Sakuri, T., Satou, M., Akiyama, K., Taji, T., Yamaguchi-Shinozaki, K., Carninci, P., Kawai, J., Hayashizaki, Y., and Shinozaki, K. (2002). Monitoring the expression profiles of 7000 Arabidopsis genes under drought, cold, and highsalinity stresses using a full-length cDNA microarray. Plant J. 31, 279-292.

Sewelam, N., Oshima, Y., Mitsuda, N., and OhmeTakagi, M. (2014). A step towards understanding plant responses to multiple environmental stresses: a genome-wide study. Plant Cell Environ. 37, 2024-35.

Shaik, R., and Ramakrishna, W. (2013). Genes and co-expression modules common to drought and bacterial stress responses in Arabidopsis and rice. PLoS ONE 8, e77261.

Sham, A., Al-Azzawi, A., Al-Ameri, S., Al-Mahmoud, B., Awwad, F., Al-Rawashdeh, A., Iratni, R., and AbuQamar, S. (2014). Transcriptome analysis reveals genes commonly induced by Botrytis cinerea infection, cold, drought and oxidative stresses in Arabidopsis. PLoS One 25, e113718.

Sham, A., Moustafa, K., Al-Ameri, S., Al-Azzawi, A., Iratni, R., and AbuQamar, S. (2015). Identification of Arabidopsis candidate genes in response to biotic and abiotic stresses using comparative microarrays. PLoS ONE 10, e0125666.
Shi, H., Qian, Y., Tan, D.X., Reiter, R.J., and He, C. (2015b). Melatonin induces the transcripts of CBF/DREB1s and their involvement in both abiotic and biotic stresses in Arabidopsis. J. Pineal. Res. 59, 334-42.

Shi, H., Tan, D.X., Reiter, R.J., Ye, T., Yang, F., and Chan, Z. (2015a). Melatonin induces class A1 heat-shock factors (HSFA1s) and their possible involvement of thermotolerance in Arabidopsis. J. Pineal. Res. 58, 335-342.

Skibbe, D.S., Doehlemann, G., Fernandes, J., and Walbot, V. (2010). Maize tumors caused by Ustilago maydis require organ-specific genes in host and pathogen. Science 328, 89-92.

Su, L.C., Deng, B., Liu, S., Li, L.M., Hu, B., Zhong, Y.T., Li, L. (2015). Isolation and characterization of an osmotic stress and ABA induced histone deacetylase in Arachishypogaea. Front. Plant Sci. 6, 512.

Suzuki, N., Rivero, R.M., Shulaev, V., Blumwald, E., and Mittler, R. (2014). Abiotic and biotic stress combinations. New Phytol. 203, 32-43.

Takasaki, H., Maruyama, K., Kidokoro, S., Ito, Y., Fujita, Y., Shinozaki, K., Yamaguchi-Shinozaki, K., and Nakashima, K. (2010). The abiotic stressresponsive NAC-type transcription factor OsNAC5 regulates stress-inducible genes and stress tolerance in rice. Mol. Genet. Genomics. 284, 173-183.

Tanaka, H., Osakabe, Y., Katsura, S., Mizuno, S., Maruyama, K., Kusakabe, K., Mizoi, J, Shinozaki, K., and Yamaguchi-Shinozaki, K. (2012). Abiotic stress-inducible receptor-like kinases negatively control ABA signaling in Arabidopsis. Plant J. 70, 599-613.

Thomma, B.P., Nürnberger, T., and Joosten, M.H. (2011). Of PAMPs and effectors: the blurred PTIETI dichotomy. Plant Cell 23, 4-15.

Trdá, L., Boutrot, F., Claverie, J., Brulé, D., Dorey, S., and Poinssot, B. (2015). Perception of pathogenic or beneficial bacteria and their evasion of host immunity: pattern recognition receptors in the frontline. Front. Plant Sci. 6, 219.

Tsuda, K., and Katagiri, F. (2010). Comparing signaling mechanisms engaged in patterntriggered and effector-triggered immunity. Curr. Opin. Plant Biol. 13, 459-465.

Tsuda, K., and Somssich, I. (2015). Transcriptional networks in plant immunity. New Phytol. 206, 932-947.

Virdi, A., Singh, S., and Singh, P. (2015). Abiotic stress responses in plants: roles of calmodulinregulated proteins. Front. Plant Sci. 6, 809.

Wang, A., Yu, X., Mao, Y., Liu, G., Liu, Y., and Niu, $X$. (2015). Overexpression of a small heat-shock- 
protein gene enhances tolerance to abiotic stresses in rice. Plant Breeding 134, 384-393.

Wang, W., Vinocur, B., Shoseyov, O., and Altman, A. (2004). Role of plant heat-shock proteins and molecular chaperones in the abiotic stress response. Trends Plant Sci. 9, 244-252.

Wang, X., Basnayake, B., Zhang, H., Li, G., Li, W., Virk, N., Mengiste, T., and Song, F. (2009). The Arabidopsis ATAF1, a NAC transcription factor, is a negative regulator of defence responses against necrotrophic fungal and bacterial pathogens. Mol. Plant Microbe Interact. 22, 1227-1238.

Wang, Y., Bao, Z., Zhu, Y., and Hua J. (2009). Analysis of temperature modulation of plant defense against biotrophic microbes. Mol. Plant Microbe Interact. 22, 498-506.

Weston, D.J., Karve, A.A., Gunter, L.E., Jawdy, S.S., Yang, X., Allen, S.M., and Wullschleger, S.D. (2011). Comparative physiology and transcriptional networks underlying the heat shock response in Populus trichocarpa, Arabidopsis thaliana, and Glycine max. Plant Cell Environ. 34, 1488-506.

Wilkins, O., Brautigam, K., and Campbell, M.M. (2010). Time of day shapes Arabidopsis drought transcriptomes. Plant J. 63, 715-727.

Wu, Y., Deng, Z., Lai, J., Zhang, Y., Yang, C., Yin, B., Zhao, Q., Li, Y., Yang, C., and Xie, Q. (2009). Dual function of Arabidopsis ATAF1 in abiotic and biotic stress responses. Cell Res. 19, 1279-1290.

Xin, M., Wang, Y., Yao, Y., Xie, C., Peng, H., Ni, Z., and Sun, Q. (2010). Diverse set of microRNAs are responsive to powdery mildew infection and heat stress in wheat. BMC Plant Biol. 10, 123.

Xue, G.P., Drenth, J., and Mcintyre, C.L. (2015). TaHsfA6f is a transcriptional activator that regulates a suite of heat stress protection genes in wheat (Triticum aestivum L.) including previously unknown Hsf targets. J. Exp. Bot. 66, 1025-1039.

Zhang, B. (2015). MicroRNA: a new target for improving plant tolerance to abiotic stress. J. Exp. Bot. 66, 1749-1761.

Zhang, F., Zhang, F., Huang, L., Vera Cruz, C., Ali, J., Xu, J., Zhou, Y., and Li, Z. (2016). Overlap between signalling pathways responsive to Xanthomonas oryzae pv. oryzae infection and drought stress in rice introgression line revealed by RNA-Seq. J. Plant Growth Regul. 35, 345-356.

Zhao, J., Gao, Y., Zhang, Z., Chen, T., Guo, W., and Zhang, T. (2013). A receptor-like kinase gene (GbRLK) from Gossypium barbadense enhances salinity and drought-stress tolerance in Arabidopsis. BMC Plant Biol. 13, 110.

Zipfel C. (2014) Plant pattern-recognition receptors. Trends in Immunology 35, 345-351.

Zou, X., Qin, Z., Zhang, C., Liu, B., Liu, J., Zhang, C., Lin, C., Li, H., and Zhao, T. (2015). Overexpression of an S-domain receptor-like kinase extracellular domain improves panicle architecture and grain yield in rice. J. Exp. Bot. 66, 7197-7209. 
